\title{
Characterizing breast lesions using functional optical imaging guided by an independent $X$-ray mammogram
}

\author{
Bin Deng, ${ }^{1, *}$, Maxim Fradkin ${ }^{2}$, Daniel B. Kopans ${ }^{3}$, David A. Boas ${ }^{1}$, Magnus Aslund ${ }^{2}$, and Qianqian Fang ${ }^{1}$ \\ ${ }^{1}$ Massachusetts General Hospital, Athinoula A. Martinos Center for Biomedical Imaging, Charlestown, MA 02129 \\ ${ }^{2}$ Philips Research Medisys, 33 rue de Verdun, Suresnes, France 92156 \\ ${ }^{3}$ Massachusetts General Hospital, Department of Radiology, Boston, MA 02114 \\ *bdeng1@nmr.mgh.harvard.edu
}

\begin{abstract}
We validated a new method combining stand-alone 2D mammogram with functional
DOT imaging. 2D-guided optical reconstruction reveals similar statistics to spatially co-registered 3D-guided reconstruction in differentiating tumor from other tissues.
\end{abstract}

(C)2014 Optical Society of America

OCIS codes: 100.3010 (Image reconstruction techniques); 170.6960 (Tomography); 170.3830 (Mammography).

\section{Introduction}

According to the American Cancer Society, breast cancer ranks the second among other principal causes of death in women. At present, clinical recommendations are focused on the early detection of breast cancer, which usually results in less extensive treatment and better outcomes. Screening techniques, ranging from self-examinations and clinical palpation to gold-standard x-ray mammographic imaging, allow the detection and removal of invasive tumor at its early stage, reducing mortality for breast cancer. However, to date, the positive predictive value of mammography is rather low, leading to unnecessary biopsies $70-80 \%$ of the time [1].

The fact that differentiating malignant and benign lesions based purely on mammographic breast images is challenging even for experienced radiologists. This is partially because mammogram is only capable of revealing structural characteristics while leaving the pivotal metabolic information of cancerous tissue behind. To address such limitation, various noninvasive, nonionizing image-contrasting methods, especially those on tissue functions, such as diffuse optical tomography (DOT), have been investigated [2,3]. Physiological parameters like total hemoglobin concentration ( $\mathrm{HbT})$, oxygen saturation $\left(\mathrm{SO}_{2}\right)$, blood flow, reduced scattering coefficients $\left(\mu_{\mathrm{s}}\right)$, water and lipids concentrations provide a window into tissue physiology. The ability to derive tissue functional parameters and its low-cost potential make DOT hold significant promise for clinical integration and translation.

We have been developing a combined three-dimensional (3D) digital breast tomosynthesis (DBT) and DOT system [4]. Despite the early success of this DOT/DBT system in our clinical studies [4], its commercial promotion is restrained by a very limited market presence of DBT. Given over 9,000 existing two-dimensional (2D) digital mammography machines installed in the US alone, an efficient implementation and rapid clinical adoption of DOT could be achieved by using DOT as a stand-alone optical imaging device in conjunction with an existing 2D mammography system. The desire of making DOT independent to the mammography system requires registrations between two separate breast compressions, one for optical and one for x-ray. Over the past two years, we have developed an efficient contour-based registration algorithm, allowing the transformation of the breast anatomical information from $\mathrm{x}$-ray to the breast geometry used for optical imaging to guide the optical reconstruction, similar to the DOT/DBT analysis [5].

The focus of this study is to clinically validate our 2D mammogram guided DOT approach using 34 clinical cases acquired over the past years. Particularly, we are interested in answering the following questions: 1) are the optical properties obtained from a 2D-guided reconstruction similar to those from a DOT/DBT analysis? 2) are the findings differentiating malignant from benign lesions of DOT/DBT approach, shown in our previous publications, still valid when using a separately acquired mammogram as priors? In the following sections, we will first describe the technical details for the data analysis procedures. This is followed by statistical analyses using a clinical population to quantitatively compare between optical images generated with $2 \mathrm{D}$ and 3D structural guidance. Lastly, we discuss the findings and areas for future improvement to achieve the proposed goals.

\section{Materials and Methods}

\section{Optical image reconstruction guided by separately acquired $2 D$ mammograms}

We use previously acquired DOT/DBT clinical measurements to quantitatively validate the new approach. For each DOT/DBT dataset, we have collected spatially co-registered optical and 3D breast anatomy. In addition, we also obtained the diagnostic mammogram (2D) acquired separately on a different machine. The 2D image was registered to a breast mesh derived from the DBT image. The details of the contour-based registration are described in [6].

To jointly consider the structural information in an optical reconstruction, we apply a prior-guided reconstruction technique, described in [5]. Briefly, we segment the 2D mammographic image into probability maps of two breast 
tissue compositions, namely adipose and fibroglandular, represented by a set of compositional vectors. Using aforementioned 2D-to-mesh contour transformation, such 2D-guided probability map is vertically extrapolated to form a volumetric image, and mapped onto the $3 \mathrm{D}$ breast mesh for the reconstruction. In addition, for each tumor case, we ask a senior radiologist to identify the centroid of the primary lesion, from which we then create a tumor probability map determined by a Gaussian-sphere profile, which has a maximum of 1 and a full-width halfmaximum of $2 \mathrm{~cm}$ for all lesions. The probability maps for the fibroglandular and adipose tissues are scaled accordingly to ensure the sum of the three components is always 1 at any given location.

To enable a pair-wised analysis between the lesioned and the contralateral healthy breast, we applied the same contour-based registration algorithm to co-register bilateral mammograms, and then mirror the tumor probability map from the tumor side to the healthy side, as shown in Fig. 1(b). Note that for these cases, no lesion actually exists in the contralateral breast. Use of a mirrored tumor prior enables us to: 1) test the tumor prior guided reconstruction performance in the case of a non-existent tumor, and 2) extract the optical properties from the bilateral breasts to form a pair-wised analysis between lesion and healthy tissues.

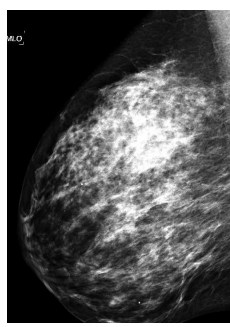

(a)

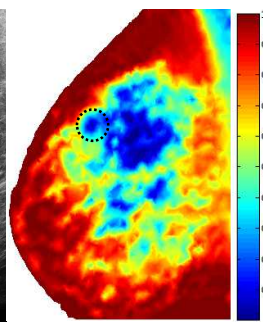

(b)

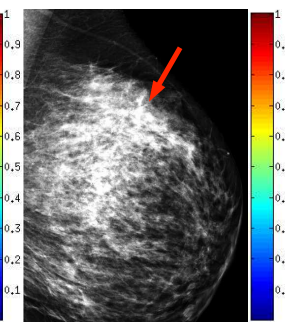

(c)

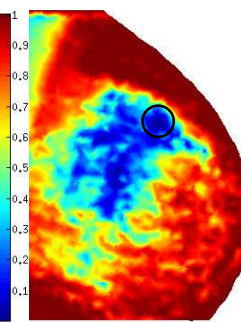

(d)

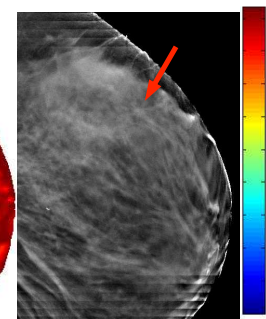

(e)

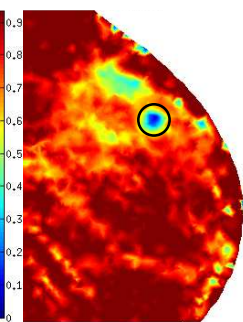

(f)

Fig. 1. 2D mammogram of a healthy breast (a) and its contralateral breast with a malignant tumor (c), followed respectively by their 2D-guided priors presented by probabilities of being adipose (b,d). For comparison, a DBT slice of tumor breast (e) and its adipose prior (f) also plotted. Red arrow: tumor centroid; Solid circle: tumor prior centroid; Dotted circle: mirrored centroid.

\section{Clinical data and analysis}

We retrospectively take a sub-population used for our DOT/DBT analysis to validate the 2D-guided DOT approach. This patient group contains 10 breasts with malignant tumors, 7 breasts with solid benign lesions, and 17 healthy breasts. To identify the potential clinical relevance of the proposed approach, we extract the optical properties for each tissue components, i.e. adipose, fibroglandular and tumor (for the healthy contralateral breasts, a "tumor" region-of-interest (ROI) is actually healthy tissue) by solving a least square equation, denoted as Eq. (4) in [5]. To compare the diagnostic power between the 2D- and 3D-guided optical image reconstructions, we perform paired two-tailed $t$-tests of various optical properties, i.e. $\mathrm{HbT}, \mathrm{SO}_{2}$, and reduced scattering coefficient $\left(\mu_{\mathrm{s}}{ }^{\prime}\right)$ at $830 \mathrm{~nm}$, between lesion, adipose, and fibroglandular tissues within the same breast for each technique, respectively. We then normalize the tumor and fibroglandular tissue optical properties by those of adipose in the same breast, and perform two-sampled $t$-tests between optical properties of malignant, solid benign and healthy tissues for both techniques.
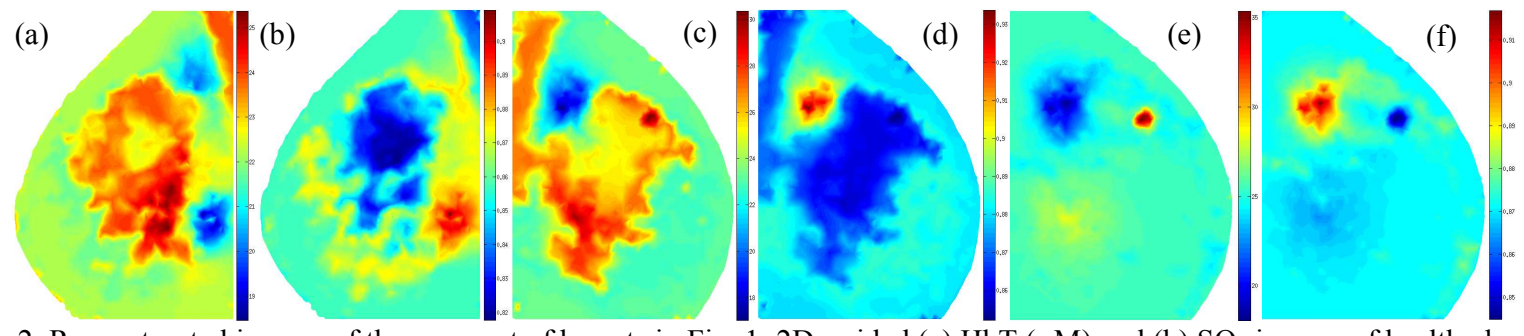

Fig. 2. Reconstructed images of the same set of breasts in Fig. 1: 2D-guided (a) $\mathrm{HbT}(\mu \mathrm{M})$ and (b) $\mathrm{SO}_{2}$ images of healthy breast; 2D-guided (c,d) and 3D-guided (e,f) images of $\mathrm{HbT}(\mu \mathrm{M})(\mathrm{c}, \mathrm{e})$ and $\mathrm{SO}_{2}(\mathrm{~d}, \mathrm{f})$, respectively, of contralateral malignant breast.

We took one step further by performing paired $t$-tests between 8 bilateral cases and identifying the statistical significance to diagnose malignant tumors using the bilateral symmetry. This was tested for both 2D and 3D guided reconstructions. Two malignant cases were excluded from this analysis due to lacking contralateral data. Statistical analyses were performed on normalized optical properties of fibroglandular tissues, and contralateral malignant tumor and healthy tissues. The priors generated using the aforementioned method guide the reconstruction as "soft" constraints. Thus, introducing a $3^{\text {rd }}$ "tumor" component in healthy breast reconstruction doesn't necessarily differentiate it from surrounding healthy tissue in optical reconstruction images, as clearly shown by comparing Fig. $2(a, b)$ with $(c, d)$. 


\section{Results}

The $p$ values from the aforementioned tests for the proposed 2D-guided image reconstruction are summarized in Table 1. Statistically significant comparisons for both 2D- and 3D-guided reconstruction are marked by "\&" and in blue bold font. Symbol "*” represents items that are significant in the 2D-guided technique but lose significance in 3D-guided reconstruction. All tests are two-tailed.

Table 1. Statistical two-tailed $t$-tests (in p-values) of optical properties of different tissue types in 2D-guided optical image reconstruction. (a) Comparisons within the same breast, grouped by lesion types; (A-Adipose; F-Fibroglandular; T-Tumor); (b) Cross group comparisons; (c) Bilateral comparisons of 8 malignant/healthy breast pairs.

\begin{tabular}{|c|c|c|c|}
\hline \multicolumn{4}{|c|}{ (a) Within the same breast } \\
\hline HbT & A vs. F & A vs. T & F vs. T \\
\hline Malignant (10) & $0.0347^{*}$ & $0.0022^{\&}$ & $0.0026^{\&}$ \\
\hline Solid Benign (8) & $0.0433^{\&}$ & 0.4024 & 0.4550 \\
\hline Healthy (17) & 0.3984 & -- & -- \\
\hline \multicolumn{4}{|c|}{$\mathrm{SO}_{2}$} \\
\hline Malignant (10) & 0.4465 & 0.9074 & 0.6581 \\
\hline Solid Benign (8) & 0.5125 & 0.9721 & 0.9121 \\
\hline Healthy (17) & 0.3694 & -- & -- \\
\hline \multicolumn{4}{|c|}{$\mu_{\mathrm{s}}^{\prime}$ at $830 \mathrm{~nm}$} \\
\hline Malignant (10) & 0.0963 & $0.0059^{\&}$ & $0.0075^{\&}$ \\
\hline Solid Benign (8) & $0.0342^{\&}$ & 0.3358 & 0.3448 \\
\hline Healthy (17) & 0.1509 & -- & -- \\
\hline
\end{tabular}

\begin{tabular}{|c|c|c|c|}
\hline \multicolumn{4}{|c|}{ (b) Cross group comparisons } \\
\hline & $\begin{array}{c}\text { Malignant vs. } \\
\text { Solid Benign }\end{array}$ & $\begin{array}{c}\text { Malignant vs. } \\
\text { Healthy }\end{array}$ & $\begin{array}{c}\text { Solid Benign } \\
\text { vs. Healthy }\end{array}$ \\
\hline HbT & $\mathbf{0 . 0 1 1 1}^{\&}$ & $\mathbf{0 . 0 0 0 3}$ & 0.7967 \\
\hline $\mathbf{S O}_{\mathbf{2}}$ & 0.9314 & 0.7739 & 0.6863 \\
\hline $\boldsymbol{\mu}_{\mathbf{s}}{ }^{\star}$ & 0.5268 & $\mathbf{0 . 0 0 1 9}^{\star}$ & 0.2664 \\
\hline
\end{tabular}

\begin{tabular}{|c|c|c|}
\hline \multicolumn{3}{|c|}{ (c) Bilateral comparisons } \\
\hline & Fibroglandular & Healthy vs. Malignant \\
\hline HbT & 0.1708 & $\mathbf{0 . 0 2 6 7}$ \\
\hline $\mathbf{S O}_{\mathbf{2}}$ & 0.2572 & 0.2101 \\
\hline $\boldsymbol{\mu}_{\mathbf{s}}{ }^{\text {( }}$ & 0.9653 & 0.0526 \\
\hline
\end{tabular}

\section{Discussion and conclusions}

As reported in Table 1(a), the proposed technique of generating healthy and tumor priors from separate 2D mammograms is successful in differentiating both adipose and fibroglandular tissue from malignant tissues for $\mathrm{HbT}$ and $\mu_{\mathrm{s}}$ ', but not for $\mathrm{SO}_{2}$. The similar patterns of statistics between the 2D- and 3D-guided reconstructions also indicate the proposed 2D-guided reconstruction preserves the statistical powers as in 3D-guided technique, thus has clinical equivalence. Though not shown in Table 1, the mean $\mathrm{HbT}$ of adipose and fibroglandular tissue of all investigated breasts are $29.46 \pm 13.67 \mu \mathrm{M}, 30.24 \pm 13.20 \mu \mathrm{M}$ respectively in $2 \mathrm{D}$-guided results, while these values are $29.32 \pm 13.88 \mu \mathrm{M}, 31.73 \pm 13.02 \mu \mathrm{M}$ in $3 \mathrm{D}$-guided scenario. Despite the apparent variance, which could be a result of limited sample size, the mean values are consistent between the two techniques. The results shown in Table 1(b) further assure us that the 2D-guided reconstructions appear to be as effective as the 3D-guided reconstructions in terms of differentiating malignant from solid benign lesions with $\mathrm{HbT}(\mathrm{p}=0.0111)$, and from the healthy (mirrored) tissues with $\mathrm{HbT}(\mathrm{p}=0.0003)$ and $\mu_{\mathrm{s}}{ }^{\prime}(\mathrm{p}=0.0019)$, similar to the DBT-guided reconstructions. With no surprise, bilateral tests, as shown in Table 1(c), of the same tissue type, i.e. fibroglandular, between healthy and lesion breasts have no significance. By contrast, same tests between healthy and contralateral malignant tumors show significance $(\mathrm{p}=0.0267)$ and near significance $(\mathrm{p}=0.0526)$ using $\mathrm{HbT}$ and $\mu_{\mathrm{s}}^{\prime}$, respectively, in 2D-guided reconstruction.

In summary, we compared the optical properties recovered based on two different techniques: spatially coregistered 3D DBT-guided reconstruction and a post-registered 2D mammogram guided reconstruction. Due to inevitable variations introduced by compression related irreproducible factors, e.g. breast orientation, tissue stress, compression strength, and etc., and the coarse approximation by simply extending 2D priors to all mesh layers, degradation of optical images is expected. Nevertheless, two techniques resemble closely in the absolute optical property values, as well as the statistical significance between different tissue types. From these tests, we believe that the proposed 2D-guided optical image reconstruction technique has comparable capabilities in differentiating lesion of various types to an integrated DOT/DBT system. As a result, the clinical potentials of this technique deserve further investigations.

\section{Acknowledgement}

The authors are grateful to the funding support from the Massachusetts Life Science Center (\#2011D000334).

\section{References}

1. Pisano E.D, et al., Retrospective cost-effectiveness analysis of screening mammography. Journal of the National Cancer Institute, 353, 177383 (2005).

2. Gibson A., Hebden J., Arridge S.R., Recent advances in diffuse optical imaging, Physics in Medicine and Biology, 50, R1 (2005).

3. Busch D.R., Choe R., et al., Towards non-invasive characterization of breast cancer ad cancer metabolism with diffuse optics, PET Clin., 8(3), 345-365 (2013).

4. Fang, Q., Selb, J., Carp, S., et al., Combined optical and X-ray tomosynthesis breast imaging, Radiology 258(1), $89-97$ (2011).

5. Fang, Q., Moore, R., Kopans, D., Boas, D., Compositional-prior-guided Image Reconstruction Algorithm for Multi-modality Imaging, Biomed. Opt. Express 1(1), 223-235 (2010).

6. Fradkin M., Hofmann M., et al., Diagnosing breast cancer using independent diffuse optical tomography and x-ray mammography scans, Proc. SPIE., 8574, 85740G (2013). 